\title{
Esteatoepatite Não Alcoólica Induzida por Rápida Perda de Peso em Uso de Balão Intragástrico - Um Relato de Caso
}

\section{RESUMO}

A obesidade é uma doença crônica, atualmente considerada uma epidemia global. Está associada a várias co-morbidades clínicas, entre elas a doença esteatótica hepática não alcoólica (DEHNA), e sua complicação, a esteatoepatite não alcoólica (EHNA). Apresentamos um caso de uma paciente de 58 anos com obesidade refratária ao tratamento clínico, submetida à colocação do balão intragástrico (BIG), que evoluiu com um quadro de esteatoepatite não alcoólica, associada a rápida perda de peso. (Arq Bras Endocrinol Metab 2007;51/4:631-634)

Descritores: Obesidade; Balão intragástrico; Esteatoepatite não alcoólica; Perda de peso

\section{ABSTRACT}

Non-Alcoholic Steatohepatitis Induced by Fast Weight Loss During the Use of Intragastric Balloon - a Case Report.

Obesity is a chronic disease that has been considered an epidemic nowadays. It is associated to much co-morbidity, such as non-alcoholic fatty liver disease (NAFLD) and its complication, steatohepatitis. We report a case of a 58-yearold obese patient refractory to clinical treatment who was submitted to the use of intragastric balloon (BIB), developing steatohepatitis induced by fast weight loss. (Arq Bras Endocrinol Metab 2007;51/4:631-634)

Keywords: Obesity; Intragastric balloon; Non-alcoholic steatohepatitis; Weight loss

\begin{abstract}
A OBESIDADE É UMA DOENÇA CRÔNICA atualmente considerada uma epidemia global, está associada a uma série de co-morbidades clínicas, dentre elas a doença esteatótica hepática não alcoólica (DEHNA) (1). Esta é a doença hepática mais comum nos Estados Unidos, acometendo 15 a $20 \%$ da população, e tem como complicação a esteatoepatite não alcoólica (EHNA) (2). Normalmente, uma substancial perda de peso tende a evoluir com a melhora significativa da esteatose hepática. Entretanto, apesar de reconhecermos a associação entre a rápida perda de peso e a ocorrência de EHNA (3), seu relato não tem sido freqüente. Reportamos um caso onde essa complicação ocorreu durante a utilização do balão intragástrico (BIG), que é mais uma possibilidade terapêutica no tratamento dos pacientes obesos. A paciente assinou um termo de consentimento livre e esclarecido autorizando a publicação do caso.
\end{abstract}

\section{RELATO DE CASO}

Paciente do sexo feminino, branca, 58 anos, do lar, com história de obesidade infanto-juvenil e vários tratamentos prévios sem sucesso, IMC de 42,3 kg/m² apresentação de caso

\author{
AleXANDer Koglin Benchimol \\ ISABELLA SILVA CARDOSO \\ JULIA FANDIÑO \\ THALITA BITTAR \\ SÍLVIA FREITAS \\ Walmir Ferreira COUtinho
}

Grupo de Obesidade e Transtornos Alimentares (GOTA) Escola Médica de PósGraduação PUC-RJ, Rio de Janeiro, RJ.

Recebido em 06/12/06

Revisado em 26/02/07

Aceito em 29/03/07 
(altura 1,60 m e peso 108,5 kg). Apresentava hipertensão arterial (HAS) e transtorno de compulsão alimentar periódico (TCAP), condições que se encontravam compensadas clinicamente com uso de valsartana (160 $\mathrm{mg} /$ dia) e fluoxetina ( $40 \mathrm{mg} /$ dia), respectivamente. Seus exames complementares mostravam esteatose hepática visualizada à ultra-sonografia abdominal, com enzimas hepáticas normais e sorologias negativas para hepatites A, B e C. Após a falência do tratamento clínico para a obesidade e considerando que a paciente não aceitava submeter-se, naquele momento, à cirurgia bariátrica, foi indicada a colocação do BIG, que seria associado ao acompanhamento multidisciplinar já realizado (endocrinologista, gastroenterologista, psiquiatra e nutricionista).

A paciente foi submetida à colocação do BIG por via endoscópica, em regime de hospital-dia, sob anestesia geral. Apresentou boa tolerância ao balão e, já no primeiro mês, obteve uma perda de $11 \mathrm{~kg}$. Nesse período, apresentou sintomas referentes ao uso do balão (pirose, náuseas e plenitude pós-prandial). Esses sintomas foram bem controlados com medicamentos sintomáticos - prócinético (bromoprida), inibidor da bomba de prótons (omeprazol) e antiespasmódico (hioscina). Ao final do primeiro mês, apresentou queixa importante de astenia de evolução contínua e progressiva; entretanto, seu exame físico mostrava-se semelhante ao exame prévio à colocação do balão. Tendo em vista a queixa de astenia, uma nova rotina laboratorial foi solicitada, incluindo enzimas hepáticas. Os resultados evidenciaram uma elevação isolada de transaminases, sem outras alterações laboratoriais (tabela 1). A conduta tomada foi a suspensão da atividade física e a redução da dose de fluoxetina para 20 $\mathrm{mg} /$ dia, com solicitação de novos exames complementares para prosseguimento da investigação diagnóstica.

No mês subseqüente, voltou para uma nova avaliação clínica com peso de $95,5 \mathrm{~kg}$ (perda de $2 \mathrm{~kg}$ ) e referindo melhora da astenia. Os resultados da avaliação laboratorial demonstraram uma diminuição das transaminases (porém ainda se mantinham discretamente elevadas) e excluíram hepatopatias virais, autoimunes e por depósito de substâncias como ferro e cobre. A paciente não apresentava história de uso de álcool, sendo então considerada a hipótese diagnóstica de EHNA induzida pela rápida perda de peso.

Nos quatro meses subseqüentes, a paciente permaneceu em acompanhamento clínico e laboratorial, evoluindo com a normalização das enzimas hepáticas. Uma nova ultrassongrafia abdominal evidenciou o fígado com aspecto normal (regressão da esteatose). Tendo em vista a sua melhora tanto clínica como laboratorial, a paciente foi liberada para reiniciar a atividade física de forma progressiva, e retornar com a dose inicial de fluoxetina $(40 \mathrm{mg} / \mathrm{dia})$.

O BIG foi retirado após 6 meses, quando a paciente apresentava peso corporal de $94 \mathrm{~kg}$ (uma perda total de $14,5 \mathrm{~kg}$ ). Desde então, apresenta-se assintomática, com exames laboratoriais normais e manutenção da perda de peso.

\section{DISCUSSĀO}

O uso do BIG é mais uma opção de tratamento da obesidade e, se bem indicado, otimiza os resultados do tratamento clínico, diminuindo os riscos e complicações da cirurgia da bariátrica (4-6). Como em todo procedimento médico, existem indicações para seu emprego, assim como limitações, contra-indicações e complicações associadas à colocação do balão (tabela 2) (4,7).

O BIG foi inicialmente utilizado em 1986, mas apresentou resultados desastrosos, em grande parte relacionados à desinsuflação precoce e a obstrução intestinal, o que restringiu seu uso a centros de pesquisa. $\mathrm{O}$ balão foi aperfeiçoado desde então, já sendo aplicado na Europa desde 1992, levando a melhores resultados em relação à perda de peso e a um número menor de complicações $(4,8)$. O atual balão de silicone é introduzido no estômago por endoscopia digestiva alta, onde é insuflado até atingir o volume médio de $500 \mathrm{ml}$ (490 $\mathrm{ml}$ de solução salina e $10 \mathrm{ml}$ de azul de metileno - a presença da coloração azul nas fezes ou urina mostra que houve perfuração e desinsuflação do balão). O seu objetivo é promover uma sensação de saciedade precoce, diminuindo o consumo de alimentos e facilitando a adap-

Tabela 1. Exames laboratoriais.

\begin{tabular}{|c|c|c|c|c|c|c|c|}
\hline Exames Evolução & $\begin{array}{l}\text { Pré- } \\
\text { BIG }\end{array}$ & $\begin{array}{l}30 \text { dias } \\
\text { Pós-BIG }\end{array}$ & $\begin{array}{l}45 \text { dias } \\
\text { Pós-BIG }\end{array}$ & $\begin{array}{l}60 \text { dias } \\
\text { Pós-BIG }\end{array}$ & $\begin{array}{l}75 \text { dias } \\
\text { Pós-BIG }\end{array}$ & $\begin{array}{l}4 \text { meses } \\
\text { Pós-BIG }\end{array}$ & $\begin{array}{l}6 \text { meses } \\
\text { Pós-BIG }\end{array}$ \\
\hline TGP U/L & 23 & 468 & 327 & 257 & 54 & 26 & 30 \\
\hline TGO U/L & 28 & 114 & 69 & 70 & 26 & 18 & 26 \\
\hline Fosfatase alcalina U/L & 60 & 113 & 102 & 80 & 84 & 165 & 120 \\
\hline gama GT U/L & 30 & 18 & 20 & 20 & 18 & 41 & 21 \\
\hline bilirrubina total mg/dl & 0,50 & 0,58 & 0,52 & 0,52 & 0,53 & 0,41 & 0,50 \\
\hline
\end{tabular}


tação a uma dieta hipocalórica associada a uma atividade física regular. O tempo de permanência com o BIG é de, no máximo, 6 meses e durante todo esse período deve ser feito um acompanhamento do paciente por uma equipe multidisciplinar. Após a retirada do balão, pode ocorrer ganho de peso, principalmente se não forem preservadas as mudanças dietéticas e atividade física (4).

No caso descrito, a paciente apresentava as seguintes indicações para o uso do BIG: IMC $>35 \mathrm{~kg} / \mathrm{m}^{2}$ e doenças associadas, além da falência ao tratamento clínico da obesidade. A paciente não apresentava contraindicações ao tratamento cirúrgico, porém não desejava, naquele momento, submeter-se à cirurgia bariátrica. Cabe a ressalva de que a paciente apresentava HAS e TCAP, muito comum na obesidade grau III. O TCAP não contra-indica o procedimento, e a paciente apresentava-se em remissão dos episódios de compulsão e em uso de fluoxetina (9).

Na literatura, há relatos de inúmeras séries de tratamento da obesidade com balão intragástrico por períodos de 4 a 6 meses. Há uma perda de, em média, $14 \mathrm{~kg}$ no peso corporal e de $5 \mathrm{~kg} / \mathrm{m}^{2}$ no IMC. O peso tende a estabilizar-se após os dois primeiros meses, quando a perda passa a ser mais lenta, e isto foi exatamente o observado no caso descrito $(4,5,10)$. A recidiva da obesidade descrita após 18 meses do procedimento está em torno de 45\% (11).

A DEHNA corresponde à doença metabólica que cursa com esteatose hepatocelular predominan- temente macrovesicular. A EHNA é o nome dado às formas de DEHNA onde há a presença de esteatose associada a graus variados de atividade necroinflamatória e fibrose, condição que possui potencial evolutivo para doença hepática avançada (cirrose) e suas complicações (12). Nos EUA, a DEHNA corresponde à doença hepática mais prevalente (em torno de 20\%), com a EHNA apresentando prevalência de 2 a $3 \%$, com evolução para cirrose em quase $20 \%$ dos casos, no período de 10 anos. A esteatose pura ocorre em 70\% dos pacientes que se apresentam 10\% acima do peso ideal e em praticamente $100 \%$ dos obesos mórbidos (2).

A DEHNA está associada à obesidade, diabetes melito tipo 2 (DM2), dislipidemia, hipotireoidismo, uso de medicamentos (corticóides, amiodarona, diltiazem, nifedipina, tamoxifeno), nutrição parenteral total e situações de rápida perda de peso: cirurgias para obesidade, caquexia, bulimia e anorexia nervosa, entre outras $(2,12,13)$.

A patogênese da EHNA ainda não é totalmente conhecida, mas há evidências de que a resistência insulínica $(\mathrm{RI})$ e peroxidação lipídica façam parte deste processo. A obesidade e o DM2 (ou mesmo a RI) contribuem aumentando a síntese e reduzindo a oxidação dos ácidos graxos livres, além de estimular a lipólise tecidual, resultando em um acúmulo progressivo de lipídios nos hepatócitos. Esse estoque pode atingir níveis tóxicos, o que gera um aumento do estresse oxidativo com formação de radicais livres,

Tabela 2. Balão intragástrico* .

\section{Indicações:}

- $\quad \mathrm{IMC}>45 \mathrm{~kg} / \mathrm{m}^{2}$ com doenças associadas, no período pré-operatório das cirurgias: bariátrica, cardiovascular, ortopédica e outras cirurgias em geral.

- $\quad \mathrm{IMC}>35 \mathrm{~kg} / \mathrm{m}^{2}$ e doenças associadas, refratárias ao tratamento clínico, mas que apresentem contra-indicações para o tratamento cirúrgico.

- IMC $<35 \mathrm{~kg} / \mathrm{m}^{2}$ com falha de tratamento clínico bem orientado (por período superior a três anos) com presença ou risco de doenças associadas, ou ainda efeitos colaterais importantes às medicações utilizadas.

\section{Contra-indicações:}

- Absolutas: cirurgia gástrica prévia, dependência de álcool ou drogas, úlcera gástrica ou duodenal em fase ativa, uso crônico de anticoagulantes, doenças do colágeno, doença inflamatória intestinal, hérnia de hiato $>5 \mathrm{~cm}$, cirrose hepática, insuficiência renal crônica, gravidez e SIDA.

- Relativas: doenças da transição gástrica potencialmente hemorrágicas, esofagite acentuada, esôfago de Barrett e uso crônico de anti-inflamatórios não-hormonais (AINES) e anticoagulante.

\section{Complicações:}

- Complicações referentes à sedação/anestesia.

- Complicações relacionadas à presença do balão intra-gástrico: náuseas e vômitos principalmente nos três dias após a colocação do balão, em até $42 \%$, com retirada precoce do balão (relato de $4 \%$ ); meteorismo e eructação ( $8 \%$ ); refluxo gastroesofágico com esofagite (12\%); úlcera péptica (1\%); desinsuflação do balão e sua expulsão pela via natural (1 a $2 \%)$ com risco de obstrução intestinal. Raramente: perfuração gástrica, dilatação gástrica crônica, deslocamento do balão com sua impactação no antro (com síndrome pilórica), além da colonização do balão por fungos.

Balão Intragástrico - gastroplastia endoscópica para o tratamento da obesidade.

Dr. José Afonso Sallet, 2001 - coordenador nacional do protocolo do balão intragástrico junto ao Ministério da Saúde, BR. 
associado a uma lesão mitocondrial. A peroxidação lipídica anormal resultará em dano hepático direto, com inflamação e até mesmo fibrose $(2,12,14,15)$.

O diagnóstico clínico da EHNA nesta paciente foi baseado na queixa de astenia durante o período de rápida perda de peso, no aumento em 5 vezes das enzimas hepáticas, na presença de componentes da síndrome metabólica (HAS, obesidade e RI), na exclusão do consumo de álcool, e de hepatopatias virais, autoimunes, genéticas ou induzidas por drogas. Isso vai de acordo com os dados de literatura $(2,12,13,16)$.

A intervenção proposta para esta paciente foi o acompanhamento clínico, suspensão da atividade física e a avaliação seriada das transaminases. Foi feita diminuição da dose de fluoxetina - visto a metabolização hepática desta droga, mas sem promover descompensação do quadro de TCAP. Houve redução progressiva das transaminases, coincidindo com a perda de peso mais lenta nos meses subseqüentes em uso do balão, até a normalização dos exames laboratoriais e melhora da astenia. Um mês após a retirada do balão, a paciente encontrava-se assintomática, com exames normais e perda de peso mantida.

A distinção entre DEHNA e EHNA não pode ser feita apenas baseada em critérios clínicos ou laboratoriais, porém o papel da biópsia hepática continua controverso $(2,13)$. A biópsia pode excluir outras causas de hepatopatias, distinguir DEHNA de EHNA, além de avaliar o grau de fibrose, podendo determinar o prognóstico da doença. Na prática clínica, a biópsia é indicada nos pacientes com fatores de risco para DEHNA/EHNA que apresentam TGP persistentemente elevada ( $\geq 1,5$ vezes o limite superior da normalidade) após tentativa de controle metabólico e/ou retirada dos fatores desencadeantes por um período máximo de 6 meses $(2,12)$. É preciso ressaltar que a biópsia hepática é um procedimento invasivo com riscos associados ao método e custos de internação, constituindo mais um argumento contra a realização da biópsia de forma rotineira. Portanto, a decisão de sua realização deve ser individualizada, baseada caso a caso (13). Sendo assim, optamos por não biopsiar esta paciente, visto a evolução benigna do caso, mantendo-a em acompanhamento clínico e laboratorial.

A esteatose hepática com alterações inflamatórias e fibrose em pacientes obesos já é conhecida há décadas. Porém, passou a ser observada como entidade clínica após os relatos de insuficiência hepática em alguns pacientes submetidos à cirurgia bariátrica com bypass jejunoileal $(13,16)$. Atualmente, novas técnicas, cirúrgicas e não cirúrgicas, para o tratamento da obesidade vêm sendo desenvolvidas. Tendo em vista a alta prevalência da obesidade na população e maior facilidade de acesso às técnicas para tratamento da mesma, é preciso atenção a essa complicação hepática, principalmente quando a intervenção proposta pode levar a uma perda de peso muito rápida, conforme observado por Andersen e cols. (17). Ressaltamos que essa complicação também pode se desenvolver com o uso do $\mathrm{BIG}$, como relatado. É preciso identificar com que freqüência isso vem ocorrendo, e qual a sua conseqüência a longo prazo, já que é um método que vem se firmando como mais uma opção no tratamento da obesidade.

\section{REFERÊNCIAS}

1. Benchimol AK, Coutinho WF. Obesidade mórbida e afecções associadas. In: Garrido Júnior $A B$ et al. Cirurgia da obesidade - Sociedade Brasileira de Cirurgia Bariátrica. Rio de Janeiro: Atheneu, 2002. pp. 13-8.

2. Yu AS, Keeffe EB. Nonalcoholic fatty liver disease. Gastroenterol Disor 2002;2(1):11-9.

3. Luyckx FH, Desaive C, Thiry A, Dewe W, Scheen AJ, Gielen $\mathrm{JE}$, et al. Liver abnormalities in severely obese subjects: effect of drastic weight loss after gastroplasty. Int J Obes Relat Metab Disord 1998;22(3):222-6.

4. Sallet JA. Gastroplastia endoscópica para o tratamento da obesidade: balão intragástrico. São Paulo: Caminho Editorial, 2001.

5. Doldi SB. Treatment of morbid obesity with intragastric balloon in association with diet. Obes Surg 2002;12(4):583-7.

6. Wahlen $\mathrm{CH}$, Bastens $\mathrm{B}$, Herve J, Malmendier C, Dallemagne $B$, Jehaes C, et al. The Bioenterics Intragastric Balloon (BIB): how to use it. Obes Surg 2001:11(4):524-7.

7. Kerrigan DD. Large bowel impaction by the Bioenterics Intragastric Balloon necessitating surgical intervention. Ann R Coll Surg Engl 2001;83(2):148.

8. Vasudeva R. Taylor intragastric ballon. Am J Gastroenterol 1990;85(12):1655-6.

9. Fandiño J, Benchimol AK, Coutinho WF, Appolinário JC. Cirurgia bariátrica: aspectos clínico-cirúrgicos e psiquiátricos. Rev Psiquiatr 2004;26(1):47-51.

10. Evans JD, Scott MH. Intragastric balloon in the treatment of patients with morbid obesity. Br J Surg 2001;88(9):1245-8.

11. Doldi SB, Micheletto G, Prisco F, Zappa MA, Lattuada E, Reitano M. Intragastric balloon in obese patients. Obes Surg 2001; 10:578-81.

12. Ferraz MLG, Silva AEB. Manual de condutas em hepatites. São Paulo: 2003.

13. Sanyal AJ; American Gastroenterological Association. AGA technical review on nonalcoholic fatty liver disease. Gastroenterol 2002;123(5):1705-25.

14. Liangpunsakul S, Chalasani N. Treatment of nonalcoholic fatty liver disease. Curr Treat Opt Gastroenterol 2003;6(6):455-63.

15. Angulo P. Nonalcoholic fatty liver disease. N Engl J Med 2002;346(16):1221-9.

16. Baker $A L$, Elson CO, Jaspan J, Boyer JL. Liver failure with steatonecrosis after jejunoileal bypass: recovery with parenteral nutrition and reanastomosis. Arch Intern Med 1979;139(3):289-92.

17. Andersen T, Gluud C, Franzmann MB, Christoffersen P. Hepatic effects of dietary weight loss in morbidly obese subjects. J Hepatol 1991;12:224-9.

\section{Endereço para correspondência:}

Alexander Koglin Benchimol

Av. Jornalista Ricardo Marinho 300 / 1204

22631-350 Rio de Janeiro, RJ

Fax: (21) 3171-1846

E-mail: alexanderbenchimol@rjnet.com.br 